\title{
Estimation and Tracking of the Power Quality Disturbances in the Frequency Domain
}

\author{
Dušan Agrež \\ Faculty of Electrical Engineering, University of Ljubljana, Trzaska 25, 1001 Ljubljana, Slovenia, dusan.agrez@fe.uni-lj.si \\ In this paper, simple algorithms for fast measurement and estimation of the unknown changing frequency, amplitude, and phase \\ difference of the sinusoidal signals from two channels with the same frequency, as well as other parameters for evaluation of the \\ power quality disturbances, are presented. Parameters are calculated from the DFT coefficients around the component peaks. The \\ improvement for reducing the influences of the harmonic components is the estimation with the three-point interpolation and the \\ Hann window.
}

Keywords: Power quality, voltage quality, disturbance estimation, frequency domain, non-coherency.

\section{INTRODUCTION}

$\mathrm{T}^{\mathrm{s}}$ he main consequence of the growth of nonlinear loads is the increase in the harmonic content of the power network, and this leads to serious power quality (PQ) issues. Harmonic pollution is one of the major concerns of voltage quality (VQ) and power quality, and many proposals for measurement of the power quality events can be found in the literature [1]-[2]. Two main categories of PQ disturbances can be introduced: variations and events [3], belonging respectively to steady state and transient phenomena. Variations can be divided into two categories defined as harmonic and interharmonic distortions, while events comprise interruptions, dips, sags, swells, and so on. Requirements for measurement instruments and methods are defined in [4]. This standard suggests the realization of a measurement instrument based on algorithms in the frequency domain.

Therefore, an accurate estimation of the power supply frequency as a basic signal parameter is required even in the presence of disturbances. Besides estimation of the frequency of the fundamental power component, the changing amplitude and phase have to be estimated to evaluate the values of the active power $P$, the apparent power $S$, and the reactive power $Q$ [5]. The problem of measurement and evaluation of the power quality disturbances can be reduced to the parameters estimation of each spectral component (frequency [7], amplitude [11]) for VQ and phase difference for PQ in the presence of the noncoherency and the noise.

\section{SIGNAL ANALYSIS AND ESTIMATIONS}

Non-stationary signals are generally multi-component $m$. The sampled version $g(k \Delta t)_{N}$ (1) is obtained in two measurement steps. The data cord is extracted from the original signal by sampling $\left(f_{\mathrm{s}}=1 / \Delta t \quad\right.$ - sampling frequency) and windowing $(w(k)$ - windowing coefficients).

$$
\begin{aligned}
g(k \Delta t)_{N} & =w(k) g(k \Delta t)_{\infty}= \\
& =\sum_{m} A_{m}(k \Delta t) \cdot \sin \left[2 \pi\left(s_{m, f} k \Delta t+f_{m}\right) k \Delta t+\varphi_{m}\right]
\end{aligned}
$$

Components have time-varying frequencies $f_{m}(t)$, amplitudes $A_{m}(t)$, and phases $\varphi_{m}(t)$. If the time of observation is short enough, we can approximate the change in frequency by a linear variation with the frequency slope $s_{m, f}$, where $f_{m}$ and $\varphi_{m}$ are the initial frequency and phase of the stationary part of the component. Due to the changing frequency and, with this, the non-coherent sampling conditions, the problem of leakage effects increases. There are several methods to improve coherence, among which the most important are: re-sampling of the signal [8], the adaptive Fourier analysis [9], the Chirplet transformation [10], and so on. This paper presents effective algorithms for fast measurement and estimation of the unknown changing frequency, amplitude, and phase difference of the signal component for tracking the power quality disturbances.

In the stationary state, the DFT [7] at the spectral line $i$ is given by

$$
G(i)=-\frac{j}{2} \sum_{m} A_{m}\left[W\left(i-\theta_{m}\right) e^{j \varphi_{m}}-W\left(i+\theta_{m}\right) e^{-j \varphi_{m}}\right]
$$

where $W(*)$ is a spectrum of the window function $w(k)$ and $\theta_{m}=f_{m} / \Delta f=i_{m}+\delta_{m}$ is a frequency divided by the frequency resolution $\Delta f=1 /(N \Delta t)$. The displacement term $\delta_{m}$ is due to the non-coherency.

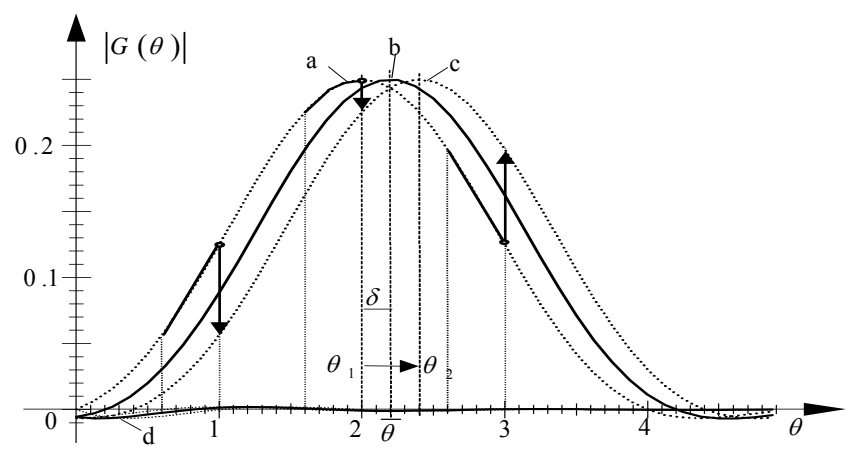

Fig. 1. Influences on the amplitude DFT coefficients when frequency is changing from $\theta_{1}=2$ (a) to $\theta_{2}=2.4$ (c) (the Hann window is used). 
In Fig. 1, the changes in the DFT coefficients when frequency is linearly changing are presented. In the local maximum of the amplitude part of the DFT, where the shortrange leakage of the window spectrum (the first part in (2)) is dominant, the coefficients on the front side - direction of the frequency movement - increase (Fig. 1: $|G(i=3)| \uparrow$ ) and those on the rear side decrease (Fig. 1: $|G(i=1)| \downarrow$ ).

The DFT coefficients are changing according to the curve of the main lobe of the window used, if the frequency is changing linearly. In the complete figure of changes in coefficients, we should also consider the long-range leakage parts of the window spectrum (the second part in (2) and curve $\mathrm{d}$ in Fig. 1.). Considering a single component and $a=\pi(N-1) / N$ in (2), this equation can be rearranged. The same expression is valid for the rectangular window and the Hann window:

$$
|G(i)|=\frac{A_{m}}{2}\left|W\left(i-\theta_{m}\right)\right| \cdot\left|1-\frac{\left|W\left(i+\theta_{m}\right)\right|}{\left|W\left(i-\theta_{m}\right)\right|} e^{-j 2\left(a \theta_{m}+\varphi_{m}\right)}\right|
$$

The long-range leakage contributions (the second part in (3)) are frequency and phase dependent. The errors of the coefficients, when frequency is changing linearly, have two parts: the bias contribution, which is a function of the window spectrum main lobe

$$
\bar{E}=E_{\text {bias }}=\frac{1}{\theta_{2}-\theta_{1}} \int_{\theta_{2}}^{\theta_{1}} W(i-\theta) \mathrm{d} \theta-W\left(i-\frac{\theta_{1}+\theta_{2}}{2}\right),
$$

and the long-range contribution with double dependence on frequency and phase. The long-range contributions drop with increasing relative frequency: $\propto 1 / \theta^{3}$ with the Hann window and $\propto 1 / \theta$ with the rectangular window.

Both contributions can be reduced by interpolation of the DFT coefficients. It has been shown [7] that the best estimation results in reducing long leakage effects are given by the three-point estimation using the Hann window. In the estimation of the particular component $m$, the three largest local DFT coefficients $\left|G\left(i_{m}-1\right)\right|,\left|G\left(i_{m}\right)\right|$, and $\left|G\left(i_{m}+1\right)\right|$ are used for frequency:

$$
{ }_{3} \delta_{m} \cong 2 \frac{\left|G\left(i_{m}+1\right)\right|-\left|G\left(i_{m}-1\right)\right|}{\left|G\left(i_{m}-1\right)\right|+2\left|G\left(i_{m}\right)\right|+\left|G\left(i_{m}+1\right)\right|},
$$

When the displacement $\delta_{m}$ for the specific component is determined, it is also possible to estimate the amplitude by summing the largest three local DFT coefficients around the signal component in the same manner as in the frequency estimation [11]:

$$
\begin{aligned}
&{ }_{3} A_{m} \cong \frac{\pi \delta_{m}}{\sin \left(\pi \delta_{m}\right)} \frac{\left(1-\delta_{m}^{2}\right)\left(4-\delta_{m}^{2}\right)}{3} . \\
& \cdot\left[\left|G\left(i_{m}-1\right)\right|+2\left|G\left(i_{m}\right)\right|+\left|G\left(i_{m}+1\right)\right|\right]
\end{aligned}
$$

The price of the effective leakage reduction is an increase in the estimation uncertainties related to the unbiased Cramér-Rao bounds fixed by the Signal-to-Noise-Ratio for the particular component $S N R_{m}=A_{m}^{2} /\left(2 \sigma_{t}^{2}\right)$ corrupted by a white noise with standard uncertainty $\sigma_{t}[6]$.

In the cases of two-channel acquisition systems for power measurements the values of phases of each channel are not required. The only value needed is in fact the phase difference $\varphi_{m, u}-\varphi_{m, i}$ of the investigated component from channel $u$ and $i$ with the same frequency $f_{m}$. The leakage effects can be reduced greatly if simultaneousness of the sampling on both channels is assumed and the measurement time of signals is the same. The assurance of these conditions gives equal displacements $\delta_{m, u}=\delta_{m, i}=\delta_{m}$, if the measurement frequency is the same $\theta_{m, u}=\theta_{m, i}=\theta_{m}$.

The component phase can be expressed as [12]:

$$
{ }_{1} \varphi_{m}=\arg \left(G\left(i_{m}\right)\right)-a \delta_{m}+\frac{\pi}{2} \mp_{1} \Delta \varphi\left(i_{m}\right)
$$

where $\Delta \varphi\left(i_{m}\right)$ is the disturbing angle due to the long-range leakage contributions. Looking for the phase difference of two signals with the same frequency, the frequency dependent part $a \delta_{m}$ can be eliminated:

$$
{ }_{1} \varphi_{u}-{ }_{1} \varphi_{i}=\arg \left(G_{u}\left(i_{m}\right)\right)-\arg \left(G_{i}\left(i_{m}\right)\right) \mp_{1} \Delta \varphi_{u}\left(i_{m}\right) \pm_{1} \Delta \varphi_{i}\left(i_{m}\right)
$$

The phase difference can be estimated by the difference in the arguments of the largest local DFT coefficients if the disturbing angles can be neglected.

$$
{ }_{1} \varphi_{u}-{ }_{1} \varphi_{i} \doteq \arg \left(G_{u}\left(i_{m}\right)\right)-\arg \left(G_{i}\left(i_{m}\right)\right)
$$

The single phase can also be estimated with the arguments of the three largest local DFT coefficients $\varphi_{i_{m}}=\arg \left[G\left(i_{m}\right)\right][12]:$

${ }_{3} \varphi_{m}=\frac{\left(1-\delta_{m}\right) \varphi_{i_{m}-1}+4 \varphi_{i_{m}}+\left(1+\delta_{m}\right) \varphi_{i_{m}+1}}{6}-\frac{2 a \delta_{m}}{3}+\frac{\pi}{2} \pm_{3} \Delta \varphi\left(i_{m}\right)$

where the disturbing angle ${ }_{3} \Delta \varphi\left(i_{m}\right)$ is much smaller than ${ }_{1} \Delta \varphi\left(i_{m}\right)$.

The phase difference can be estimated only with weighted phase differences of the largest local DFT coefficients of both signals.

$$
{ }_{3} \varphi_{u}-{ }_{3} \varphi_{i} \doteq \frac{1}{6}\left(\left(\varphi_{u, i_{m}-1}-\varphi_{i, i_{m}-1}\right)+4\left(\varphi_{u, i_{m}}-\varphi_{i, i_{m}}\right)+\left(\varphi_{u, i_{m}+1}-\varphi_{i, i_{m}+1}\right)\right)
$$

Decreasing the systematic errors increases the noise error contribution. The Cramér-Rao (CR) lower bound for the phase difference [13] is

$$
\sigma_{\mathrm{CRB}}(\Delta \varphi) / \operatorname{deg}=\frac{180}{\pi} \sqrt{\frac{S N R_{u}+S N R_{i}}{N S N R_{u} S N R_{i}}}
$$

The CR bound is achieved with the rectangular window under almost coherent sampling conditions but considering together systematic and noise error contributions the Hann window gives better results. 


\section{RESULTS OF ESTIMATIONS}

The proposed algorithms using the Hann window in the frequency domain were tested by real signals [2], and the results have been compared with the time domain estimation with the lowest achievable noise, the four-parametric sinefitting maximum likelihood estimation (MLE) [14]. For this estimation nine iterations have been used with the frequency position of the largest local DFT coefficient as the initial frequency value.

For the estimation of the harmonic distortion, the onephase voltage signal has been used with the amplitude normalization to $U=230 \mathrm{~V}$ (Fig. 2). The voltage signal was sampled with sampling frequency $f_{\mathrm{s}}=50 \mathrm{kHz}$. So, in one period we have $N=1000$ points. From a very large amount of samples the samples of 16 periods have been taken into investigation, where parameters of the signal change significantly.

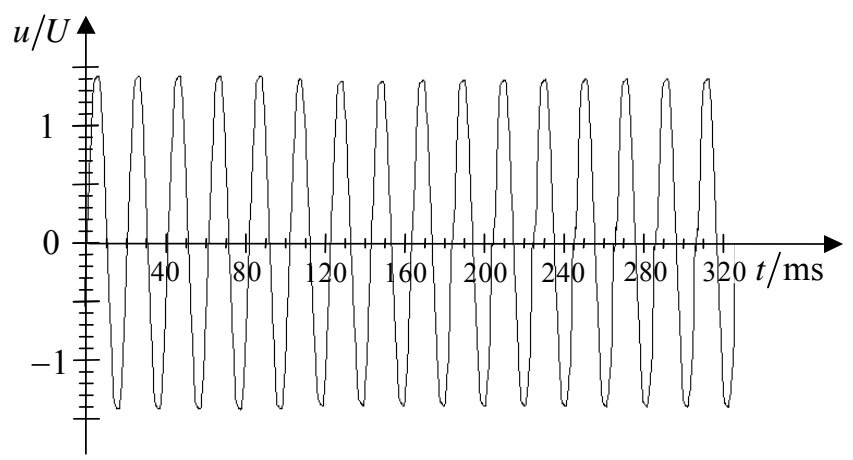

Fig. 2. Distortion of the one-phase voltage signal

First, the frequency of the fundamental component and the absolute value of the error $E(f)=f_{1, \text { est. }}-50 \mathrm{~Hz}$ were estimated (Fig. 3). The ten periods-window $\theta \approx 10$ as proposed in [4] was used. The estimated value of frequency is related to the central point of the window. The window has been moved along the time axis and we can notice very smooth estimation of the frequency variation by the interpolated DFT method (5) in comparison to the fourparametric sine-fitting method.

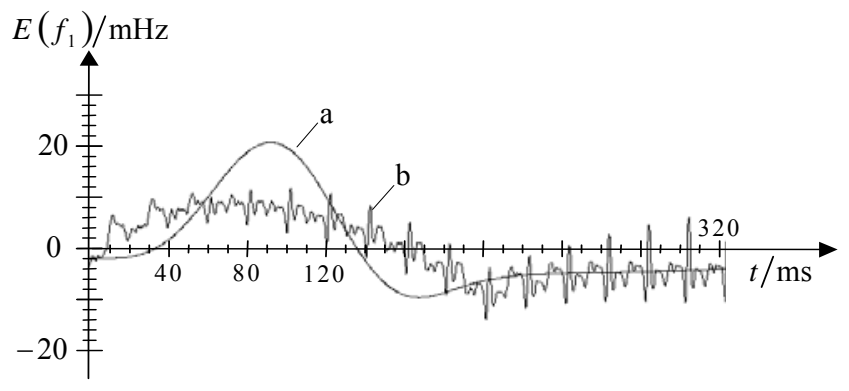

Fig. 3. Error of the frequency estimation $E\left(f_{1}\right) / \mathrm{mHz}$ : a estimation by the three-point estimation; $b$ - estimation by the four-parametric sine-fitting method

To improve the dynamic of tracking the frequency, the measurement window has to be shortened (Fig. 4.). The window can be shortened only to the three periods $\theta=3$ if the second harmonic component is presented in the signal due to the width of the Hann window main lobe. We can effectively track the frequency with the three-point estimation but some small waves can be noticed due to the closest second harmonic distortion component (Fig. 4. curve c).

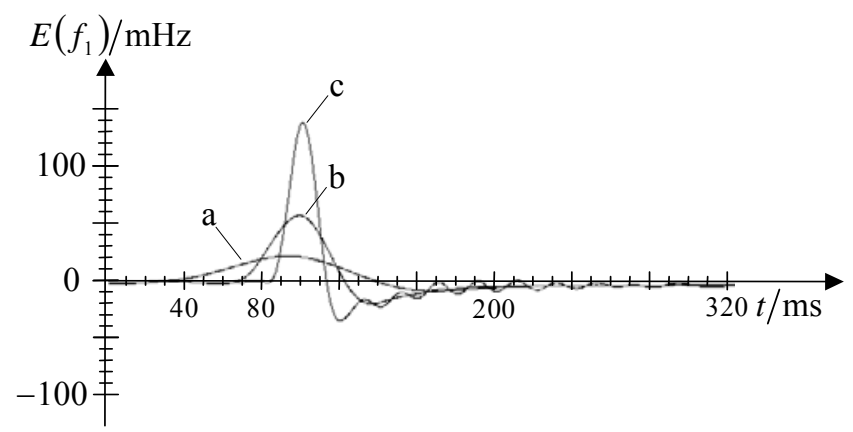

Fig. 4. Error of the frequency estimation by the three-point estimation: $\mathrm{a}-\theta=10 ; \mathrm{b}-\theta=5 ; \mathrm{c}-\theta=3$

The estimation of the amplitude of the fundamental component by the three-point estimation (6) was performed on the basis of the estimated frequency (5) and was compared to the four-parametric sine-fitting method for the amplitude (Fig. 5).

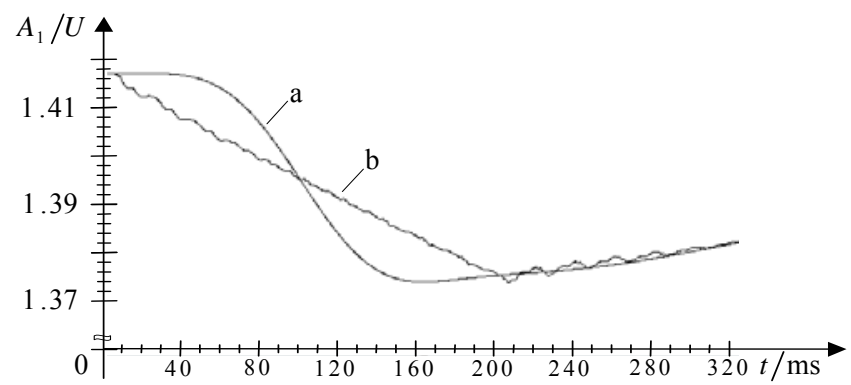

Fig. 5. The amplitude estimation of the fundamental component $A_{1} / U: \mathrm{a}$ - estimation by the three-point estimation; $\mathrm{b}$ estimation by the four-parametric sine-fitting method

If the measurement interval is shortened (Fig. 6) we can notice the same behaviors of the estimations as in the case of the frequency estimations.

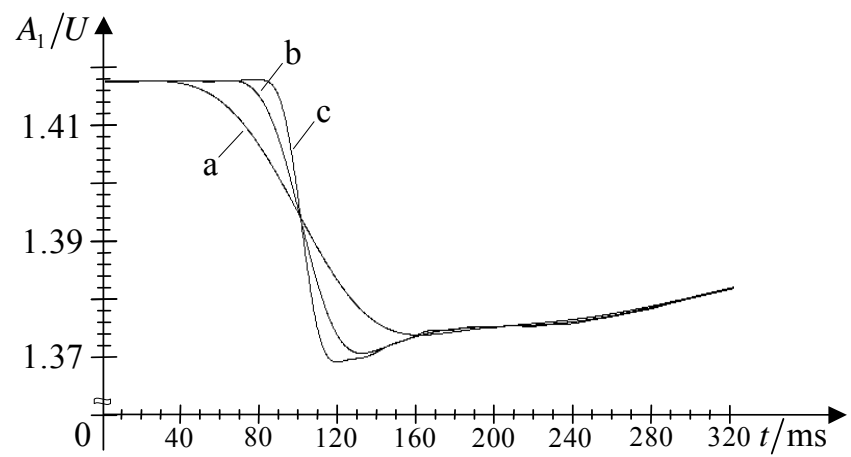

Fig. 6. The amplitude estimation of the fundamental component $A_{1} / U$ by the three-point estimation: $\mathrm{a}-\theta=10 ; \mathrm{b}-$ $\theta=5 ; \mathrm{c}-\theta=3$ 
The interpolated DFT method is also very useful for estimation of the effective or RMS value of the signal since the estimation by the definition

$$
U_{\mathrm{rms}}=\sqrt{\frac{1}{T} \int_{0}^{T} u^{2} \mathrm{~d} t}
$$

has an error in the case of the non-coherency ( $T=T_{\text {meas. }} \neq \times 1 / f_{1}$ ). The leakage error can be reduced by the interpolation in the frequency domain. We can compare the values of the $U_{\text {rms }}$ estimation in the time domain by (13) and in the frequency domain (Fig. 7) where the effective value of signal was evaluated by $U_{\mathrm{rms}}=\sqrt{\sum_{m=1}^{M}\left(A_{m} / \sqrt{2}\right)^{2}}$. The amplitudes of all harmonic components $m=1, \ldots, M$ are estimated on the basis of wellknown frequencies of these harmonics $f_{m}=m \cdot f_{1}$ by the three-point interpolation of the DFT coefficients.

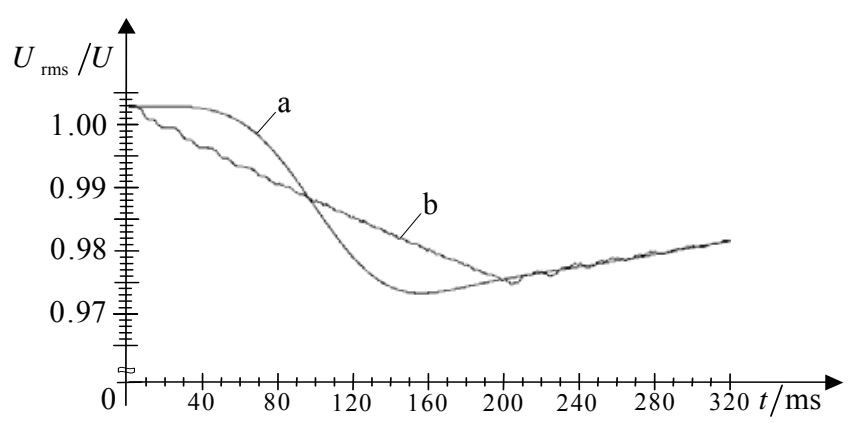

Fig. 7. RMS value of the signal: a - estimation in the frequency domain; $b$ - estimation by (13)

Improvements can also be noticed in the phase estimations of the fundamental component (Fig. 8), where the error of estimation $E\left(\varphi_{1}\right)=\varphi_{1 \text {,est. }}-\left(\varphi_{1}(t=0)+2 \pi t \cdot 50 \mathrm{~Hz}\right)$ was related to the first point at $t=0 \mathrm{~ms}$ and then the reference value of the phase was increased with time.

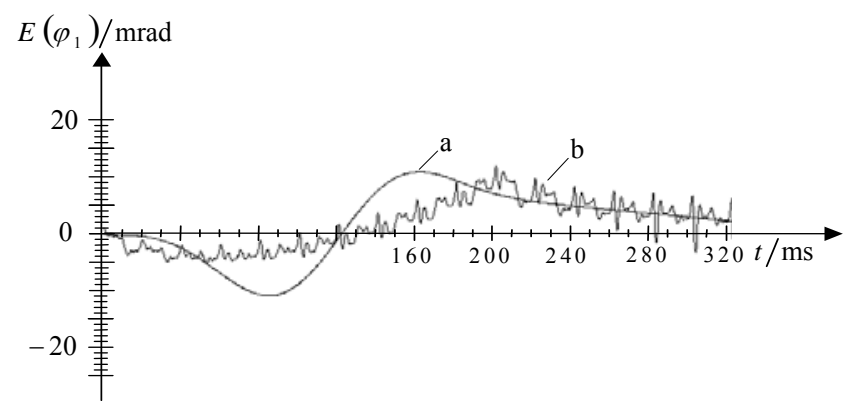

Fig. 8. Error of the phase estimation $E\left(\varphi_{1}\right) / \mathrm{rad}: \mathrm{a}$ - estimation by the three-point estimation; $\mathrm{b}$ - estimation by the four-parametric sine-fitting method

The proposed algorithms were also tested for power estimations ( $\mathrm{P}, \mathrm{S}, \mathrm{Q})$ when voltage and current signals suddenly change (Fig. 9).

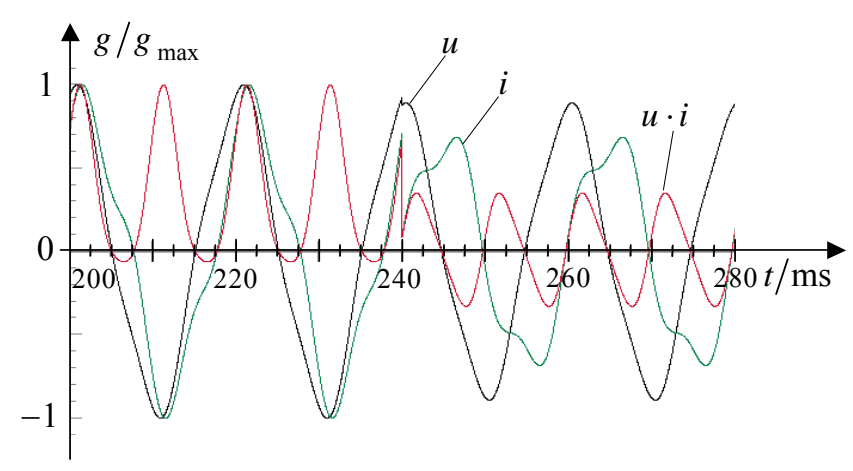

Fig. 9. Voltage, current, and instantaneous power of the one-phase load. Amplitudes of the signals $g$ are normalized to their peak values.

The two-component voltage and current signals were simultaneously sampled with frequency $f_{\mathrm{s}}=50 \mathrm{kHz}$. At time instant $t=240 \mathrm{~ms}$, the signals parameters were changed. Before the change, the signals have the following parameters:

$u_{\mathrm{b}} / \mathrm{V}=100 \cdot \sin \left(2 \pi f_{\mathrm{b}} t+\pi / 2\right)+10 \cdot \sin \left(6 \pi f_{\mathrm{b}} t+\pi / 6\right)$,

$i_{\mathrm{b}} / \mathrm{A}=5 \cdot \sin \left(2 \pi f_{\mathrm{b}} t+\pi / 3\right)+1 \cdot \sin \left(6 \pi f_{\mathrm{b}} t+\pi / 4\right), f_{\mathrm{b}}=49.9 \mathrm{~Hz}$

with values of the active power:

$P=\sum_{m} U_{m} I_{m} \cos \varphi_{m}=U_{1} I_{1} \cos \varphi_{1}+U_{3} I_{3} \cos \varphi_{3}=221,34 \mathrm{~W}$

$\left(\varphi_{m}=\varphi_{U, m}-\varphi_{I, m}\right)$,

the apparent power:

$S_{\mathrm{b}}=U I=\sqrt{U_{1}^{2}+U_{3}^{2}} \cdot \sqrt{I_{1}^{2}+I_{3}^{2}}=256,22 \mathrm{VA}$,

and the 'pure reactive' power

$Q_{\mathrm{b}}=\sum_{m} U_{m} I_{m} \sin \varphi_{m}=U_{1} I_{1} \sin \varphi_{1}+U_{3} I_{3} \sin \varphi_{3}=123,71 \mathrm{VAr}$

After the change, the signals have the following new parameters:

$u_{\mathrm{a}} / \mathrm{V}=90 \cdot \sin \left(2 \pi f_{\mathrm{a}} t+\pi / 2\right)+8 \cdot \sin \left(6 \pi f_{\mathrm{a}} t+\pi / 6\right)$,

$i_{\mathrm{a}} / \mathrm{A}=4 \cdot \sin \left(2 \pi f_{\mathrm{a}} t+0\right)+1 \cdot \sin \left(6 \pi f_{\mathrm{a}} t+\pi / 4\right), \quad f_{\mathrm{a}}=50.1 \mathrm{~Hz}$

with the new values of powers:

$P_{\mathrm{a}}=3.09 \mathrm{~W}, S_{\mathrm{a}}=184.29 \mathrm{VA}$, and $Q_{\mathrm{a}}=179.17 \mathrm{VAr}$.

Before and after the signals steps, there are non-coherent sampling conditions if we take approximately ten periods in the measurement interval as suggested by the standard [4]: $f_{\mathrm{b}}=49.9 \mathrm{~Hz} \rightarrow \theta_{\mathrm{b}}=f_{\mathrm{b}} \cdot 10000 / f_{\mathrm{s}}=9.98 \quad$ and $f_{\mathrm{a}}=50.1 \mathrm{~Hz} \rightarrow \theta_{\mathrm{a}}=10.02$.

The estimation of the active power by the definition

$$
P=\frac{1}{T} \int_{0}^{T} u i \mathrm{~d} t, \quad P=\frac{1}{N} \sum_{k=0}^{N-1} u(k) \cdot i(k), k=0, \ldots, N-1
$$

has an error in the case of non-coherency ( $\left.T=T_{\text {meas. }}=N \Delta t \neq \times 1 / f_{1}\right)$. The leakage error can be reduced by interpolation in the frequency domain if we estimate the amplitudes by (6) and the phase differences by (9) of the significant components. 


$$
P=\sum_{m} U_{m} I_{m} \cos \varphi_{m}, \quad \varphi_{m}=\varphi_{U, m}-\varphi_{I, m}
$$

If we have periodic signals the amplitudes and the phase differences of all harmonic components $m=1, \ldots, M$ are estimated on the basis of well-known frequencies of these harmonics $f_{m}=m \cdot f_{1}$.

The same is valid for the 'pure reactive' power (16) and also for the apparent power (17):

$$
\begin{aligned}
& Q=\sum_{m} U_{m} I_{m} \sin \varphi_{m}, \quad \varphi_{m}=\varphi_{U, m}-\varphi_{I, m} \\
& S=U I=\sqrt{\sum_{m=1}^{M}\left(A_{m}(u) / \sqrt{2}\right)^{2}} \cdot \sqrt{\sum_{m=1}^{M}\left(A_{m}(i) / \sqrt{2}\right)^{2}}
\end{aligned}
$$

When the window of $N=10000$ sampling points (ten signal periods) has been moved along the time axis, the active and the apparent powers have been decreased and the 'pure reactive' power has been increased (Figs. 10 I, II, III).
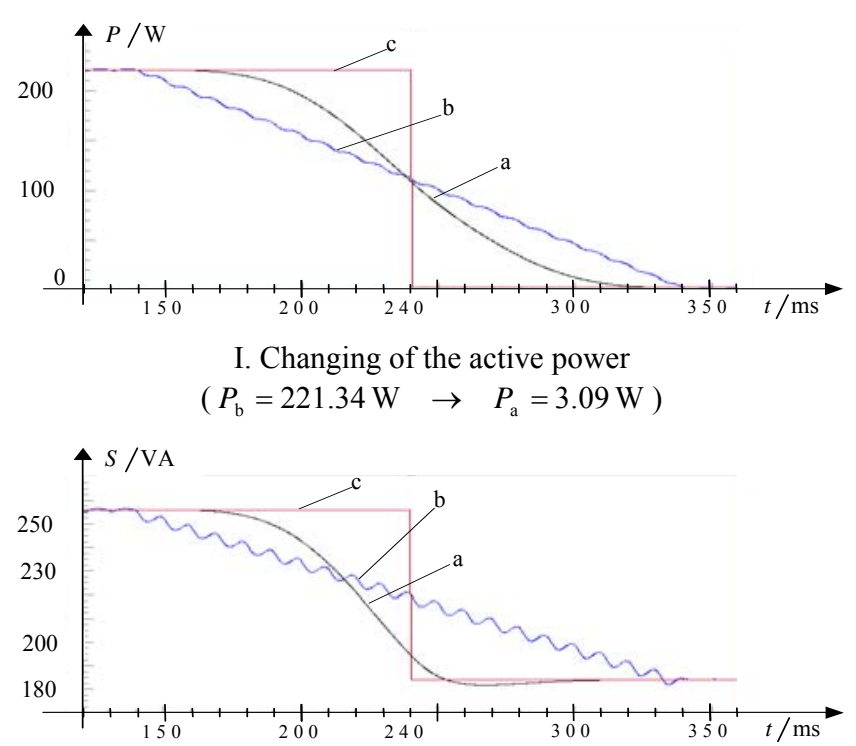

II. Changing of the apparent power $\left(S_{\mathrm{b}}=256.22 \mathrm{VA} \rightarrow S_{\mathrm{a}}=184.29 \mathrm{VA}\right)$

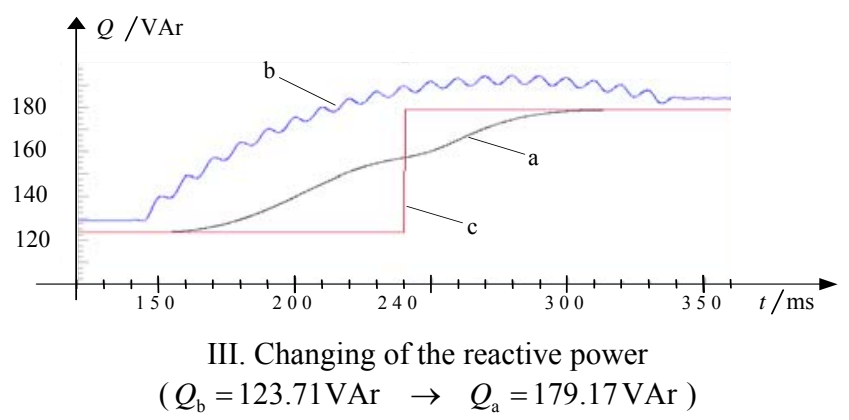

Fig. 10. Changing estimations of the powers: a - estimation in the frequency domain by IDFT; $b$ - estimation in the time domain ((14) for $P,(13)$ for $S$, and for $\left.Q=\sqrt{S^{2}-P^{2}}\right)$; c - the reference value

Again, we can notice very smooth estimations of the power variations by the interpolated DFT method using Equations (5), (6), (9), (15), (16), and (17) in comparison to the classical estimation methods by (13) and (14) and much closer to the step-like changes of the signals (Figs. 11 I, II, III).
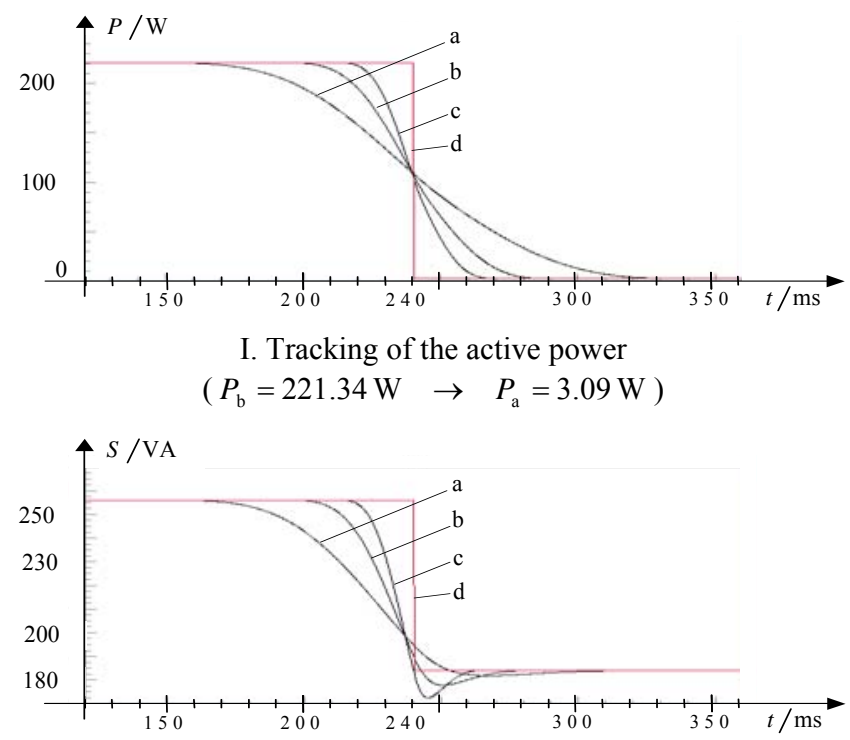

II. Tracking of the apparent power

$\left(S_{\mathrm{b}}=256.22 \mathrm{VA} \rightarrow S_{\mathrm{a}}=184.29 \mathrm{VA}\right)$

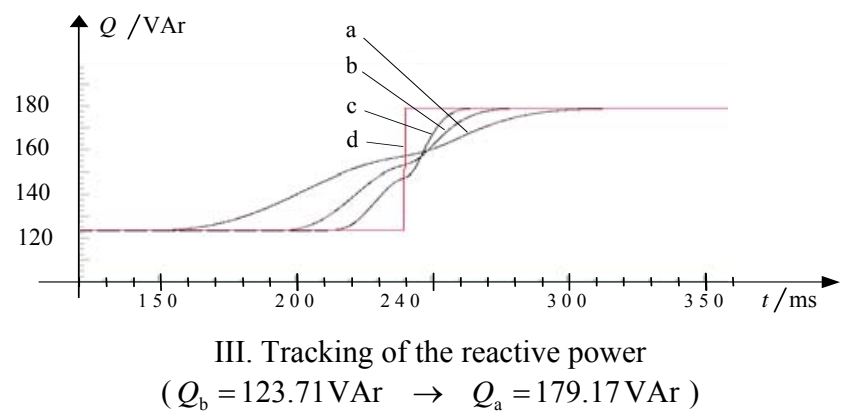

Fig. 11. Changing estimations of the powers in the frequency domain by the three-point IDFT: $\mathrm{a}-\theta=10 ; \mathrm{b}-\theta=5 ; \mathrm{c}-\theta=3$; $\mathrm{d}$ - the reference value

\section{CONCLUSION}

In this paper, simple algorithms for fast measurement and estimation of the unknown changing frequency, amplitude, phase difference, and other parameters for evaluation of the power quality disturbances are presented. Parameters are calculated from the DFT coefficients around the component peaks. Whenever disturbances are present in the power systems, at least the frequency variations can be taken into consideration and the non-coherent measurement conditions appear. In all examples of signal parameters estimation and tracking, very good behavior of the three-point estimations can be noticed and the measurement time can be reduced to the three cycles of the power signals.

\section{REFERENCES}

[1] IEEE Standard 1459-2000 (2003). IEEE Standard Definitions for the Measurement of Electric Power Quantities Under Sinusoidal, Nonsinusoidal, 
Balanced or Unbalanced Conditions. The Institute of Electrical and Electronic Engineers. Inc. New York.

[2] Matz V., Radil T., Ramos P., Serra A. C. (2007, May). Automated Power Quality Monitoring System for On-line Detection and Classifications of Disturbances. Proceedings of the IEEE IMTC/2007, no. IM-7083 - 6p., Warsaw, Poland.

[3] Bollen M. H. J. (1999). Understanding Power Quality Problems: Voltage Sags and Interruptions, IEEE Press. New York.

[4] International standard IEC 61000-4-7, 'Electromagnetic Compatibility-Testing and Measurement Techniques - General Guide on Harmonics and Interharmonics Measurements and instrumentation, for Power Supply Systems and Equipment Connected thereto', 2002.

[5] Gallo D., Landi C., Pasquino N., Polese N. (2007, October). A New Methodological Approach to Quality Assurance of Energy Meters Under Nonsinusoidal Conditions. IEEE Trans. on Instr. and Meas. 56(5), 1694-1702.

[6] Agrež D. (2010, September). Estimation of the Power Quality Disturbances in the Frequency Domain. Proceedings of the $17^{\text {th }}$ Symposium IMEKO TC 4, $3^{\text {rd }}$ Symposium IMEKO TC 19 and $15^{\text {th }}$ IWADC Workshop, no. 26, 6 p., Kosice, Slovakia.

[7] Agrež D. (2007, December). Dynamics of frequency estimation in the frequency domain. IEEE Trans. on Instr. and Meas. 56(6), 2111-2118.
[8] Backmutsky V., Zmudikov V., Agizim A., Vaisman G. (1996). A new DSP method for precise dynamic measurement of the actual power-line frequency and its data acquisition applications. Measurement 18(3), 169-176.

[9] Varkonyi Koczy A. R., Simon G., Sujbert L., Fek M. (1998, October). Fast Filter-bank for Adaptive Fourier Analysis. IEEE Trans. on Instr. and Meas. 47(5), 1124-1128.

[10] Angrisani L., D’Arco M. (2002, August). A Measurement Method Based on a Modified Version of the Chirplet Transform for Instantaneous Frequency Estimation. IEEE Trans. on Instr. and Meas. 51(4), 704-711.

[11] Agrež D. (2002). Weighted Multi-Point Interpolated DFT to Improve Amplitude Estimation of MultiFrequency Signal. IEEE Trans. on Instr. and Meas. 51, 287-292.

[12] Agrež D. (2005, August). Improving phase estimation with leakage minimization. IEEE Trans. on Instr. and Meas. 54(4), 1347-1353.

[13] Händel P. (2008, August). Parameter estimation employing a dual-channel sine-wave model under a Gaussian assumption, IEEE Trans. Instrum. Meas. 57(8), 1661-1669.

[14] Deyst J. P., Sounders T. M., Salomon O. M. (1995, June). Bounds on Least-Squares Four-Parameter SineFit Errors Due to Harmonic Distortion and Noise. IEEE Trans. on Instr. and Meas. 44, 637-642.

Received November 03, 2010. Accepted December 30, 2010. 